\title{
The Peregrine Breather on the Zero- Background Limit as the Two-Soliton Degenerate Solution: An Experimental Study
}

\section{OPEN ACCESS}

Edited by:

Jürgen Vollmer,

Universität Leipzig, Germany

Reviewed by:

Oreste Piro,

University of the Balearic Islands,

Spain

Reinaldo Roberto Rosa, National Institute of Space Research (INPE), Brazil

*Correspondence:

Amin Chabchoub chabchoub.amin.8w@kyoto-u.ac.jp

Specialty section:

This article was submitted to Interdisciplinary Physics,

a section of the journal

Frontiers in Physics

Received: 25 November 2020

Accepted: 28 July 2021

Published: 25 August 2021

Citation:

Chabchoub A, Slunyaev A, Hoffmann N, Dias F, Kibler B, Genty G, Dudley JM and Akhmediev N (2021) The Peregrine Breather on the ZeroBackground Limit as the Two-Soliton

Degenerate Solution: An

Experimental Study.

Front. Phys. 9:633549.

doi: 10.3389/fphy.2021.633549

\begin{abstract}
Amin Chabchoub ${ }^{1,2,3 *}$, Alexey Slunyaev ${ }^{4,5}$, Norbert Hoffmann ${ }^{6,7}$, Frederic Dias $^{8,9}$, Bertrand Kibler ${ }^{10}$, Goëry Genty ${ }^{11}$, John M. Dudley ${ }^{12}$ and Nail Akhmediev ${ }^{13}$

${ }^{1}$ Hakubi Center for Advanced Research, Kyoto University, Kyoto, Japan, ${ }^{2}$ Disaster Prevention Research Institute, Kyoto University, Kyoto, Japan, ${ }^{3}$ Center for Wind, Waves and Water, School of Civil Engineering, The University of Sydney, Sydney, NSW, Australia, ${ }^{4}$ Department of Nonlinear Geophysical Processes, Institute of Applied Physics RAS, Nizhny Novgorod, Russia, ${ }^{5}$ Laboratory of Dynamical Systems and Applications, National Research University Higher School of Economics, Nizhny Novgorod, Russia, ${ }^{6}$ Dynamics Group, Hamburg University of Technology, Hamburg, Germany, 'Department of Mechanical Engineering Imperial College London, London, United Kingdom, ${ }^{8}$ School of Mathematics and Statistics, University College Dublin, Dublin, Ireland, ${ }^{9}$ ENS Paris-Saclay, CNRS, Centre Borelli, Université Paris-Saclay, Gif-sur-Yvette, France, ${ }^{10}$ Laboratoire Interdisciplinaire Carnot de Bourgogne (ICB), UMR 6303 CNRS-Université Bourgogne Franche-Comté, Dijon, France,

${ }^{11}$ Photonics Laboratory, Physics Unit, Tampere University, Tampere, Finland, ${ }^{12}$ Institut FEMTO-ST, Université Bourgogne Franche-Comté CNRS UMR 6174, Besançon, France, ${ }^{13}$ Department of Theoretical Physics, Research School of Physics, The Australian National University, Canberra, ACT, Australia
\end{abstract}

Solitons are coherent structures that describe the nonlinear evolution of wave localizations in hydrodynamics, optics, plasma and Bose-Einstein condensates. While the Peregrine breather is known to amplify a single localized perturbation of a carrier wave of finite amplitude by a factor of three, there is a counterpart solution on zero background known as the degenerate two-soliton which also leads to high amplitude maxima. In this study, we report several observations of such multi-soliton with doubly-localized peaks in a water wave flume. The data collected in this experiment confirm the distinctive attainment of wave amplification by a factor of two in good agreement with the dynamics of the nonlinear Schrödinger equation solution. Advanced numerical simulations solving the problem of nonlinear free water surface boundary conditions of an ideal fluid quantify the physical limitations of the degenerate two-soliton in hydrodynamics.

Keywords: degenerate soliton, Peregrine breather on the zero-background limit, rogue waves, nonlinear waves, wave hydrodynamics

\section{INTRODUCTION}

The Peregrine breather $(\mathrm{PB})[1]$ is a fundamental wave envelope solution of the nonlinear Schrödinger equation (NLSE) localized both in space and time, yielding a three-fold amplification of the initial amplitude at the point of maximum localization. These unique characteristics have led the PB to be generally considered as a potential backbone model allowing to describe the emergence of extreme events in several physical systems $[2,3]$. Although the PB existence was originally predicted in the early eighties [1], it took about 3 decades to observe this particular wave envelope in a laboratory environment [4-6]. These initial studies have attracted significant attention and led to many follow-up studies related to PB dynamics and its peculiar physical properties [7-14]. The initial or boundary conditions 
leading to the $\mathrm{PB}$ excitation require to impose a small perturbation on top of a plane wave background. Recently, generic features of $\mathrm{PB}$ dynamics on a stationary dnoidal background have been presented [15]. The regular background solution represents only one limiting case of the exact family of NLSE dnoidal solutions while the other limit is the envelope soliton on zero-background [16, 17]. This allows a more general construction of Peregrine-type coherent structures on different type of stationary backgrounds. The respective evolution in time and space can be described by an exact solution [18].

In this paper, we experimentally investigate the $\mathrm{PB}$ dynamics in the zero background limit, which can be also associated with the degenerate case of two soliton interaction, resulting in an amplitude amplification factor of two at the point of maximum localization [19]. The laboratory experiments, conducted in different water wave flumes, are in excellent agreement with the theory when the carrier steepness is moderate. We recall that the carrier wave steepness, being physically the product of wavenumber and wave amplitude and mathematically the expansion parameter in the weakly nonlinear approximation of the water wave problem [3], is a nonlinearity indicator of wave field. Otherwise, deviations from the symmetric envelope shapes are inevitable due to the physical limitations of the NLSE approach to describe broadband and highly nonlinear processes in water waves. The numerical simulations based on the higher-order spectral method (HOSM), which accurately solves the nonlinear water wave problem, quantify the limitations in the evolution of the hydrodynamic degenerate soliton on the water surface. The HOSM results predict a recurrent focusing behavior, not anticipated by the NLSE, when the carrier wave steepness is substantial. We believe that our results will have a significant impact in nonlinear dynamics as well as integrable systems and improve fundamental understanding of extreme wave formation in a variety of nonlinear media.

\section{Higher-Order Solitons on Zero Background and the Case of Degeneracy}

The NLSE for surface gravity waves is the simplest nonlinear evolution equation that takes into account the interplay between dispersion and nonlinearity in the evolution of a narrowband wave field. Assuming unidirectional propagation of the wave field in infinite water depth, the wave envelope evolution equation reads [20]

$$
i\left(\frac{\partial \psi}{\partial t}+c_{g} \frac{\partial \psi}{\partial x}\right)+\frac{\omega}{8 k^{2}} \frac{\partial^{2} \psi}{\partial x^{2}}+\frac{\omega k^{2}}{2}|\psi|^{2} \psi=0,
$$

where $\psi(x, t)$ is the complex wave envelope, $x$ is the spatial coordinate along the wave propagation, and $t$ represents time. The parameters $\omega$ and $k$ are the carrier cyclic wave frequency and wavenumber, respectively. The latter are constrained by the gravitational acceleration $g$-dependent deep-water dispersion equation

$$
\omega^{2}=g k,
$$

and the envelope is assumed to propagate with the group velocity $c_{g}=\frac{\partial \omega}{\partial k}=\frac{\omega}{2 k}$.

The NLSE is a partial differential equation that belongs to the family of integrable evolution equations [21]. Its exact solutions provide physically relevant models for investigating the dynamics of nonlinear coherent wave groups in controlled laboratory environments. The fields of its application are hydrodynamics, optics and Bose-Einstein condensates. It is common to use the dimensionless form of Eq. 1 for simplicity, particularly when aiming for the derivation of exact solutions,

$$
i \frac{\partial \Psi}{\partial T}+\frac{\partial^{2} \Psi}{\partial X^{2}}+2|\Psi|^{2} \Psi=0,
$$

which is obtained by introducing the following transformations

$$
X=2 k\left(x-c_{g} t\right), \quad T=\frac{\omega}{2} t, \quad \Psi=\frac{k}{\sqrt{2}} \psi .
$$

One of the most-fundamental solutions of the NLSE is an isolated and stationary sech-shape nonlinear wave group on zerobackground known as envelope soliton, which can be considered as a mode of a nonlinear system remaining unchanged with propagation [22]. At the same time, interactions and collisions between envelope solitons are elastic [23, 24]. The number of solitons contained in a localized initial condition remains fixed during the follow up evolution. The zero-velocity soliton solution with an amplitude of one can be written as

$$
\Psi_{S}(X, T)=\operatorname{sech}(X) \exp (i T) .
$$

The initial-value problem for the NLSE can be solved with the help of the inverse scattering technique (IST) [21, 25, 26]. More complex (higher-order) structures containing multiple solitons can be also constructed using the Darboux transformation [27] or dressing method [28]. Each envelope soliton in these superpositions is unambiguously characterized by the pair of its two key parameters: the amplitude and the velocity. The NLSE solution describing the dynamics of two envelope solitons with fixed amplitudes 0.5 and 1.5, zero-velocities and located at the same position $X=0$, is known as the Satsuma-Yajima breather [29]

$$
\Psi_{S_{2}}(X, T)=4 \frac{\cosh 3 X+3 \cosh X \exp 8 i T}{\cosh 4 X+4 \cosh 2 X+3 \cos 8 T} \exp (i T) .
$$

This solution is periodic in $T$ and can be used for pulse nonlinear wave group compression. At $T=0$, this solution takes the form of a soliton with twice the amplitude of a single soliton of the same width, i.e. $\Psi_{S_{2}}(X, 0)=2 \operatorname{sech}(X)=2 \Psi_{S}(X, 0)$. However, this initial condition changes with propagation and evolves towards a self-compression, i.e., breathing process [29]. Such solutions also play a key role in the formation of significant irreversible spectral broadening and the creation of supercontinua as a result of soliton fission [30, 31]. Generally, when the parameters of the two envelope solitons become close, the distance between them increases and they repel each other, moving away towards infinity. Due to this fact, for more than 

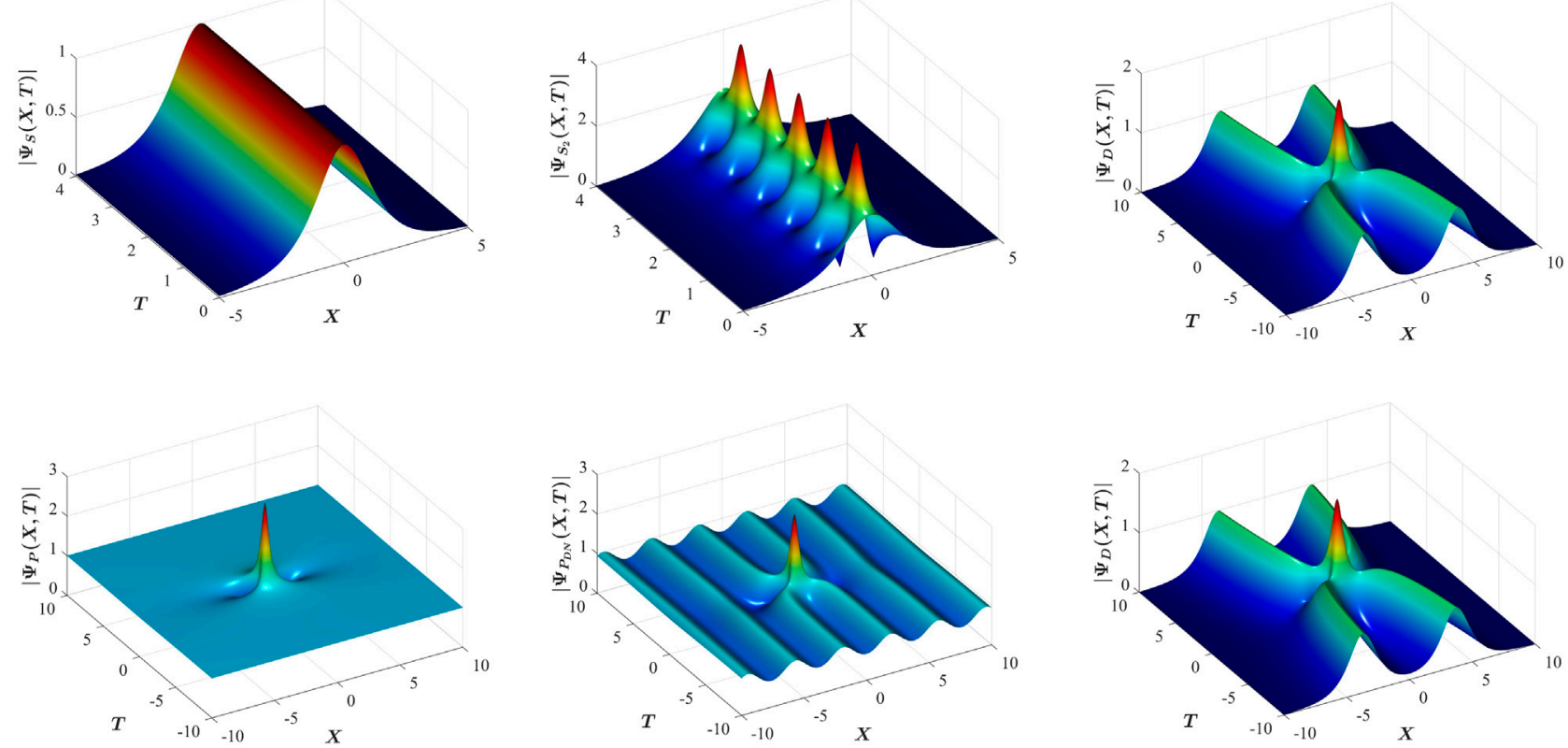

FIGURE 1 | Spatio-temporal evolution of solitons on finite and zero-background. Top left: single envelope soliton. Top middle: Higher-order soliton of order 2. Top right: Degenerate two-solution solution. Bottom left: Peregrine breather. Bottom middle: Peregrine breather on a dnoidal background. Bottom right: Degenerate two-soliton solution.

2 decades since the development of the IST, the two-soliton solution of the NLSE with exactly the same parameters has been considered as non-existent. Overcoming this controversy, the solution has been reported in $[1,19]$. Such solution is the degenerate two-soliton solution, as finding it requires considering the special limit when their amplitudes and velocities tend to the same limiting values. It is represented by a mixed semi-rational semi-hyperbolic functions

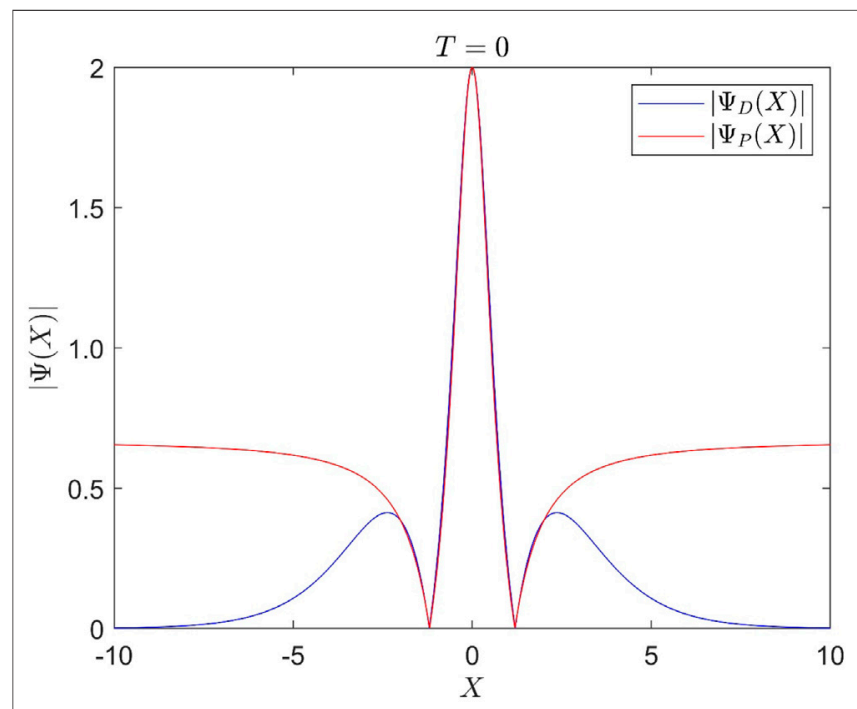

FIGURE 2 | The amplitude rescaled Peregrine breather envelope shape vs. degenerate two-soliton solution profile at $T=0$.

$$
\Psi_{D}(X, T)=4 \frac{X \sinh X-\cosh X-2 i T \cosh X}{\cosh 2 X+1+2 X^{2}+8 T^{2}} \exp (i T)
$$

Here, the subindex $D$ refers to the degenerate case. More specifically, the solution (Eq. 7) describes the interaction of two envelope solitons with unit amplitudes and with their center of mass located at $X=0$. The envelope $\left|\Psi_{D}\right|$ in (Eq. 7) is symmetric with respect to the change of the sign of either $X$ or $T$. Note that the solution (Eq. 7) may be generalized using the invariant transforms of the NLSE, i.e., arbitrary phase, scaling and Galilean transforms. In the reduced form (Eq. 7), it does not contain any free parameters. The degenerate solution (Eq. 7) describes two "attracting" envelope solitons when $T<0$. When $T=0$, the two solitons are superimposed and form an extreme event with an amplitude at the point of collision twice that the amplitude of the isolated solitons. At large times $T \gg 1$, the solution (Eq. 7) describes the two envelope solitons which slowly walk away from each other after the collision. Each of them can be approximated as a quasi-single-soliton solution. The opposite velocities of the two solitons reduce when $T \rightarrow \infty$.

What at first sight seems to be just a mathematical artifact has in fact a particular physical relevance. Indeed, the central part of the degenerate solution (Eq. 7) can be considered as the PB on the zerobackground limit. The comparison is relevant because the solution (Eq. 7) is semi-rational while PB is a rational solution. Representing hyperbolic functions $\cosh X$ and $\cosh 2 X$ in the central part of the solution as an expanded series in $X$ can reduce it to a rational approximation similar to the $\mathrm{PB}$. On the other hand, the $\mathrm{PB}$ can be excited on top of exact dnoidal solutions, parameterized as 
$\Psi_{\mathrm{dn}}(X, T)=\operatorname{dn}(X, m) \exp \left(i\left[2-m^{2}\right] T\right) ; 0 \leq m \leq 1$ see $[15,18]$. Such one-parameter family of modulated wave envelopes is steady and the parameter $m$ determines two edge cases [16, 17]. One limiting case of this one-parameter family of stationary dnoidal solutions is the regular background $(m=0)$ and the other limit is the envelope soliton $(m=1)$. This second limit leads to the formation of the degenerate soliton solution. The transformation is controlled by an additional free parameter-modulus of the dn function. The role of this parameter in the highly nontrivial process of degenerate soliton formation can be seen from Figure 7 in [15]. The latter process admits several stages of $\mathrm{PB}$ transformation. A significantly simplified version of the process can be seen from Figure 1 (see bottom panels from left to right). Here, the classical Peregrine solution on finite background is transformed to the degenerate solution on zero background with one intermediate step in the form of the $\mathrm{PB}$ on the modulated DN-wave background (referring to the semicircle in the $\lambda$-plane in Figure 2 of [15]).

The Peregrine Solution

$$
\Psi_{P}(x, t)=\left(-1+\frac{4+16 i T}{1+4 X^{2}+16 T^{2}}\right) \exp (2 i T)
$$

has been found to be present in multi-soliton solutions [32]. On the other hand, the degenerate two-soliton solution (Eq. 7) can represent Peregrine-type dynamics with zero condensate. This becomes more evident when considering the type of localization around the point of maximum amplitude. In fact, the shape of the extreme wave at $T=0$ does resemble the shape of the Peregrine breather.

This can be seen from Figure 2, where the degenerate twosoliton solution at $T=0$ is compared with the shape of the Peregrine breather at $T=0$ multiplied by the factor $2 / 3$ in order to equalize the maximal amplitudes.

The agreement between the two profiles is remarkably good within the interval between the zeros.

Even though the dynamics of the degenerate two-soliton solution creates a smaller wave amplification than the Peregrine breather (2 rather than 3 ), it is still a rapidly forming extreme event. We should also take into account the difference between the backgrounds. Thus, such solutions can be responsible for the occurrence of extreme wave events, which are very similar to the $\mathrm{PB}$.

\section{LABORATORY EXPERIMENTS}

The physical experiments have been conducted in two different water wave facilities: Hamburg University of Technology and the University of Sydney flumes, as described in [7, 33], respectively. Although both facilities are different when considering their size and type of wave generators (flap- and piston-type, respectively), the experimental procedures are similar. The wave generator is programmed to create the temporal surface elevation as described by the NLSE solution at fixed position $x^{*}$ to first-order in steepness

$$
\eta_{\text {wave maker }}\left(x^{\star}, t\right)=\operatorname{Re}\left(\psi\left(x^{*}, t\right) \exp \left[i\left(\omega t-k x^{*}\right)\right]\right) .
$$

Note that wave makers are calibrated by means of a transfer function, which is specific for each type of wave maker, to properly generate the carrier wave amplitudes and frequency as specified by Eq. 9 and any other pre-defined time-series. Since the maximal compression occurs at $x^{*}=0$, a negative value for $x^{*}$ has to be chosen in order to observe the nonlinear soliton interaction and the gradual breather-type focusing process in the wave facility. The larger $\left|x^{\star}\right|$, the more the two solitons move away from each other. The second-order Stokes correction is considered when comparing the collected data with the theoretical NLSE predictions at the respective gauge location $x_{g}^{*}$, that is

$$
\begin{aligned}
\eta\left(x_{g}^{*}, t\right)= & \operatorname{Re}\left(\psi\left(x_{g}^{*}, t\right) \exp \left[i\left(\omega t-k x_{g}^{*}\right)\right]\right. \\
& \left.+\frac{1}{2} k \psi^{2}\left(x_{g}^{*}, t\right) \exp \left[2 i\left(\omega t-k x_{g}^{*}\right)\right]\right) .
\end{aligned}
$$

Note also that when programming the wave maker to produce the surface elevation to first-order in steepness according to (9), results are expected to be identical as the bound waves (higher-order Stokes harmonics) are immediately generated within half a wavelength due to the intrinsic feature of the nonlinearity in the description of water waves. Moreover, fixing two key physical parameters, namely wave amplitude $a$ and the carrier frequency $f=\frac{\omega}{2 \pi}$ are sufficient to determine all physical features of the surface elevation. The choice for the specific values of the carrier amplitude and frequency is restricted to the stroke and frequency range specifications of the wave generator. The wave steepness $k a$, which is an indicator for the nonlinearity of the carrier wave, can be easily determined using





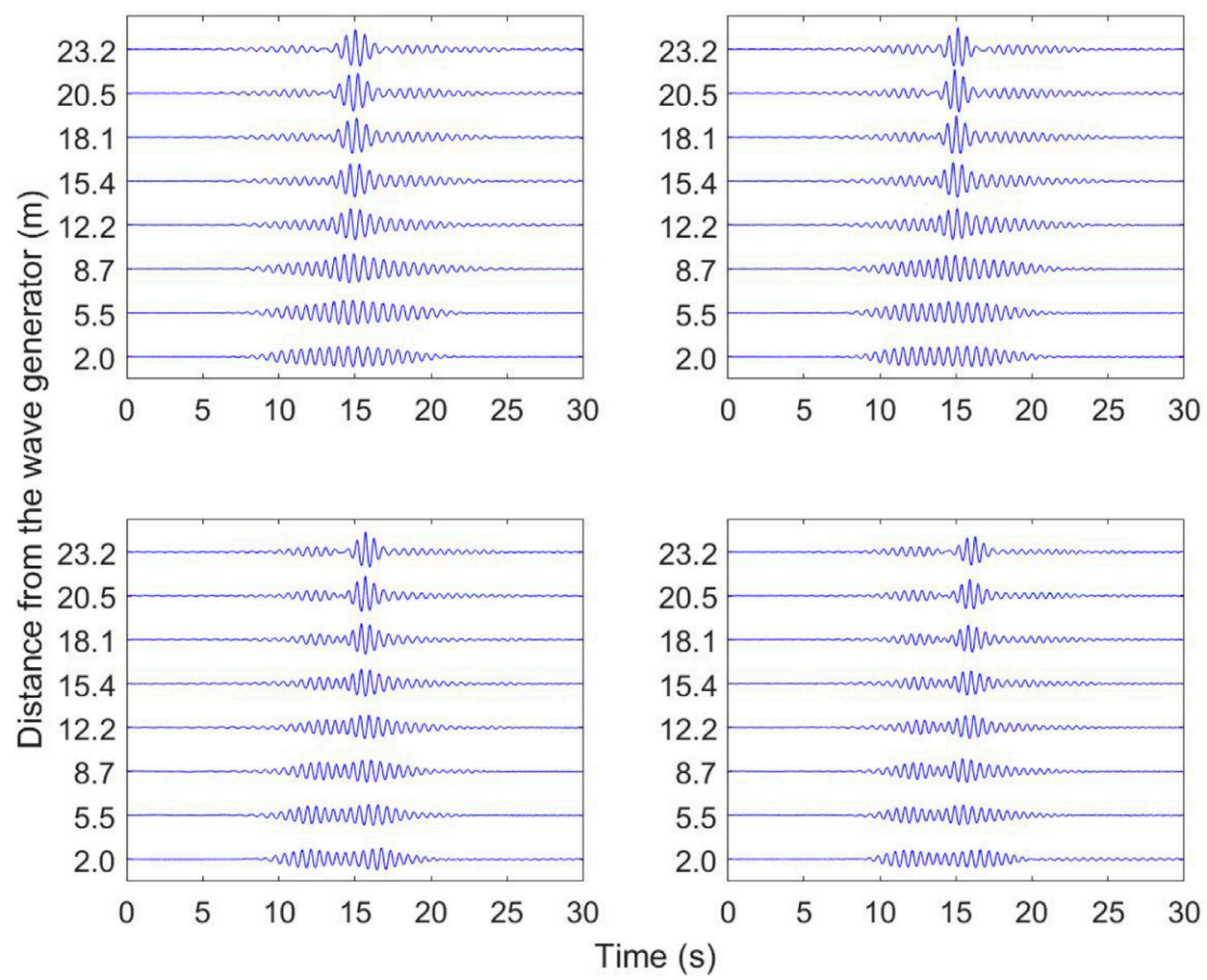

FIGURE 4 | Experimental observation of a degenerate soliton for $a=0.01 \mathrm{~m}$ and $x^{*}=-23 \mathrm{~m}$ with varying steepness values as measured in the University of Sydney flume. Top left: $k a=0.10$. Top right: $k a=0.11$. Bottom left: $k a=0.12$. Bottom right: $k a=0.13$.

the dispersion relation (Eq. 2). One crucial step consists in scaling the solution $\Psi(X, T)$ to a dimensional form $\psi(x, t)$ satisfying Eq. $\mathbf{1}$. Considering a scaling with respect to the space- or time-NLSE does not have a major impact on the evolution of the degenerate solution in a water wave flume [34]. Finally, capacitance wave gauges are installed along the flume to collect the temporal variation of the water surface at different locations in the direction of wave propagation. Note that a wave-absorbing installation is placed opposite to the wave maker to ensure a wave field propagation free of reflections. Exact schematics of both facilities can be found in $[5,33]$. A graphical guide to better understand the origin of the data as measured by the wave gauges along the flume can be found in [35].

The first experiment reported here aims to demonstrate the evolution of the solution over a significantly large distance of $45 \mathrm{~m}$ in order to observe the nonlinear and solution-specific interaction between the two envelope solitons yielding an extreme localization. On the other hand, the evolution in the Hamburg University of Technology flume was restricted to $15 \mathrm{~m}$ (when taking out the beach installation, effectively $12 \mathrm{~m}$ ). To overcome this limitation, the reflection-free wave measurement at $9 \mathrm{~m}$ was re-injected to the wave generator four times mimicking continuation of the wave propagation. The results of these tests are shown in Figure 3.

These results are a clear confirmation of degenerate soliton dynamics on the water surface. Note the excellent agreement in the distinct dynamics with the theoretical prediction, especially considering the total evolution distance of about 144 times the value of the wavelength.
There are obvious limitations of the NLSE model for water waves [36-38]. In fact, when waves become steep, the spectral broadening reduces the ability of the NLSE to accurately describe the wave hydrodynamics. However, this strongly depends on the initial carrier steepness and the bandwidth of the wave train [39].

The next series of tests have been conducted at the University of Sydney wave flume. These addressed the role of wave steepness on the collision process. Several tests have been conducted by gradually increasing the wave steepness from 0.10 to 0.13 with a 0.01 step for the same carrier amplitude of $0.01 \mathrm{~m}$. The four examples of evolution of the degenerate solution at different steepness values are shown in Figure 4.

We can clearly notice that the increase of carrier steepness distorts the clean and ideal evolution of the NLSE solution, particularly when the carrier steepness values exceed 0.13 in these laboratory tests. Consequently, the soliton interaction becomes asymmetric with a distortion of the envelope shape at the peak.

These restrictions can be accurately addressed and quantified numerically by solving the Euler equations as is discussed in the next section.

\section{NUMERICAL SIMULATIONS}

The numerical simulation is performed within the framework of the potential Euler equations using the High-Order Spectral Method (HOSM) following [40]. The HOSM simulations include $2^{10}$ grid 
points in the physical space and a twice larger number in the Fourier domain. The iterations in time are performed with the help of a split-step Fourier procedure. The order of nonlinearity is set to $M=6$. This corresponds to the solution that is accurate of up to 7wave nonlinear interactions. The initial-value problem is solved in a periodic spatial domain. The wave steepness is the only physical parameter which controls the wave evolution. The steepness is determined by the quantity $k a$, where $a$ is the amplitude of the envelope solitons long before they start to collide.

With the purpose of comparing the results of the simulations with the NLSE solution (Eq. 7), the computed surface evolution was transformed to the co-moving dimensionless variables (Eq. 4) as used in the NLSE. It is then re-scaled to provide the unit amplitudes of the envelope solitons when these are detached at $T \rightarrow-\infty$, according to the transformations similar to (Eq. 4)

$$
X=2 a k\left(x-c_{g} t\right), \quad T=a^{2} \frac{\omega}{2} t, \quad \Psi=\frac{k}{\sqrt{2}} \frac{\eta}{a}
$$

Note that in Eq. 11 the function $\Psi(X, T)$ is now real-valued. Three cases of the wave steepness were simulated, which correspond to $k a=0.05, k a=0.10$ and $k a=0.15$. In all these cases, the initial condition is specified according to the solution (Eq. 8). The dimensionless time is chosen to be $T_{0} \approx-12$. This choice corresponds to the situation when solitons already exhibit partial overlap as can be seen in Figure 5. This overlap seeds the interaction process in the simulations.

The physical time of the start of the simulation $t_{0}$ depends on the wave steepness, see Eq. 11. In fact, it corresponds to about 340 wave periods in the steepest case shown in the right panel of Figure 5, and to about 3,000 periods in the small-amplitude case shown in the left panel of Figure 5. In order to initiate the simulation of the HOSM code, the surface displacement and the surface velocity potential are calculated from $\psi\left(x,-t_{0}\right)$ with a more precise definition than Eq. 10, using the third-order asymptotic solution for nonlinear modulated waves, see [41]. Only the cases without wave breaking were simulated, thus, no filters are required to take into account wave breaking effects.

Six runs of the numerical simulations were performed with different complex phases of the initial condition $\psi\left(x,-t_{0}\right)$, for several wave steepness conditions. The envelope $\Psi_{\text {env }}(X, T)$ is calculated as the maximal values of $\Psi$ among these six simulations at every $X$ and $T$. The surface displacements of the initial conditions are plotted in Figure 5 with respect to two versions of the dimensionless space and amplitude variables.

A false color representation of the evolution of each degenerate soliton envelope in time and space is shown in Figure 6.

The intersection of the white dashed lines corresponds to the point in time and space where the maximum wave is expected within the NLSE framework. Qualitatively, the evolution of waves with small steepness $k a=0.05$ (see Figure 6 left panel) is similar to the one obtained from the NLSE theory (Figure 1 right panel) and in the laboratory experiment (Figure 3). In the simulations, the two solitary groups separated initially collide, form an extreme event and then separate again restoring their soliton shape. However, the strongly nonlinear simulation results in faster propagation of the wave groups and slightly quicker formation of the large wave (yellow dot). Interestingly, the amplitudes of the solitons after the separation are slightly different: the amplitude of the leading group is larger. The described features of the strongly nonlinear simulation become more pronounced when the steepness is larger than $k a=0.1$ (Figure 6, middle panel).

Indeed, when the steepness further increases, $k a=0.15$, the new recurrence effects are becoming more apparent (Figure 6 right panel). Moreover, when the two soliton groups merge, they form a bound state similar to the bi-soliton described in [1, 29]. However, in contrast to the bi-soliton, the interaction here is asymmetric. The two subsequent extreme events are still large in amplitude in this type of recurrent dynamics. Figure 7 shows the time evolution of the maxima of the wave elevation for the three simulations shown in Figure 6.

After a few beating cycles, the solitary groups finally decouple. At the end of the interaction process the leading soliton has a higher amplitude than the trailing one. After the three collisions, the envelope solitons are completely separated. The groups emerged after the third collision are not stationary. The leading soliton reveals the breathing dynamics (this can be seen in Figure 7 for $T>20$ ). The second solitary group spreads decaying in amplitude. Thus, the water wave dynamics of very steep degenerate solitons shows the survival of only one (leading) soliton. Its amplitude increases while the energy of the other group reduces.


FIGURE 5 | Initial conditions for the numerical simulations of the degenerate soliton. Left panel: $k a=0.05$. Middle panel: $k a=0.1$. Right panel: $k a=0.15$. The axes show the physical scaled coordinate and surface displacement ( $y$-axis left), the standard NSLE coordinate $X=k\left(x-c_{g} t\right)(x$-axis) and the complex amplitude $\Psi=\frac{k}{\sqrt{2}} \frac{\eta}{a}(y$-axis right) 

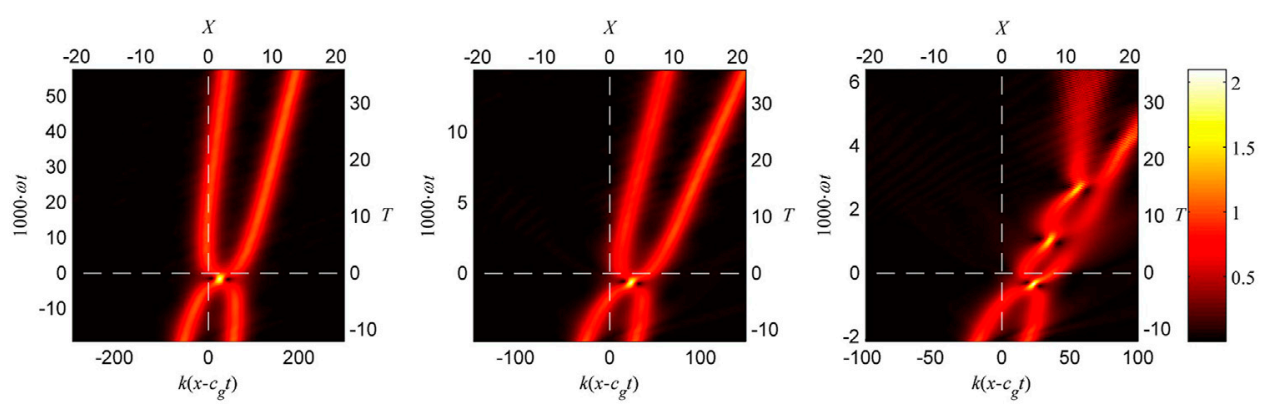

FIGURE 6 | Numerical simulations of the Euler equations of the degenerate soliton for different steepness values of the initial condition: $k a=0.05$ on the left panel, $k a=0.1$ on the middle panel, and $k a=0.15$ on the right panel. The color-coded evolution of the wave envelope $\Psi_{\text {env }}$ is shown.



FIGURE 7 | Evolution of the wave maxima in the numerical simulations shown in Figure 6.

The extreme wave groups with highest amplitude which arise in the course of the wave dynamics are shown in Figure 8.

In contrast to the envelopes shown in Figure 5, these wave profiles possess strong back-to-front asymmetry. Some alteration of the central feature with maximal amplitude, when the wave steepness grows, may be noticed as well. This difference from the experimental observations is most-probably caused by dissipative effects [17]. The maximum water elevation is slightly smaller than anticipated by the NLSE solution for $\Psi=2$ (marked by the dotted red lines in Figure 8) in the smaller wave steepness case, shown in Figure 8 (left panel). However, this limit is slightly exceeded in the case of larger wave steepness shown in Figure 8 right panel. The wave groups in Figure 8 possess noticeable vertical asymmetry in the steeper cases (Figure $\mathbf{8}$ middle and right panels) due to the bound (phase-locked) waves. While the wave crest exceeds the value of $\Psi=2$ in the steepest wave case (Figure 8 right panel), the deepest wave trough is well under the level of the NLSE solution. We emphasize that the wavelength of the carrier wave is assumed to be sufficiently large so that the capillary effects may be neglected while being small enough to satisfy the deep-water condition. We also report the evolution of the normalized and spatial Fourier transform of the three simulated evolutions, i.e., $k a=0.05, k a=0.1$ and $k a=0.15$, in Figure 9 to complete the picture.

The evolution of the Fourier modes confirms the characteristic evolution features of the degenerate two-soliton when the steepness is $k a=0.05$, thus, being very small. This appears to be the case for $k a$ $=0.1$. On the other hand, deviations start to occur with the gradual increase of carrier wave steepness. For $k a=0.15$ a noticeable beating, i.e. recurrent breathing process, can be noted.

\section{CONCLUSION}

We have reported for the first time the experimental observation of the degenerate soliton interaction in nonlinear physics. This coherent structure can be considered to be a PB on the zero-background limit. The experimental data and numerical simulations are both in excellent agreement for small and moderate carrier wave steepness values while deviations in form of beatings are observed for strongly nonlinear regimes. This fact confirms the accuracy of the
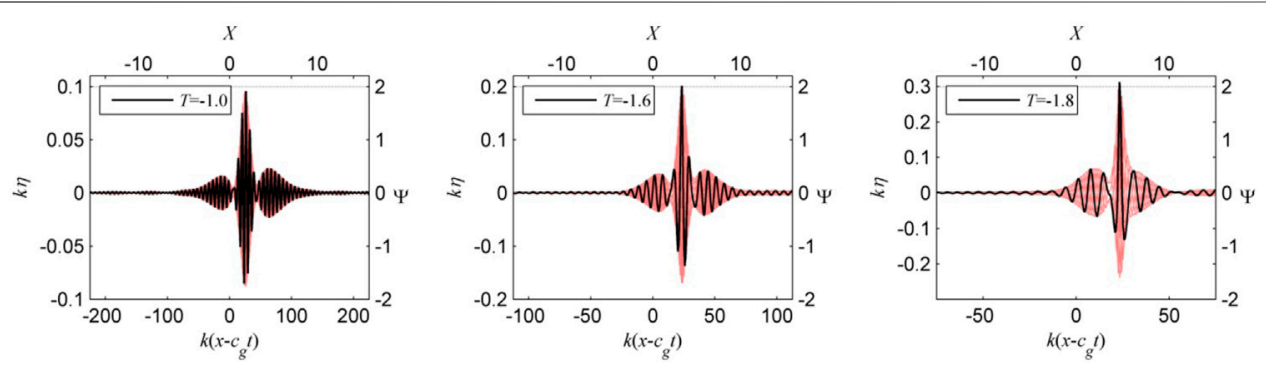

FIGURE 8 | Extreme events with highest amplitude during the evolution presented in Figure 6. 


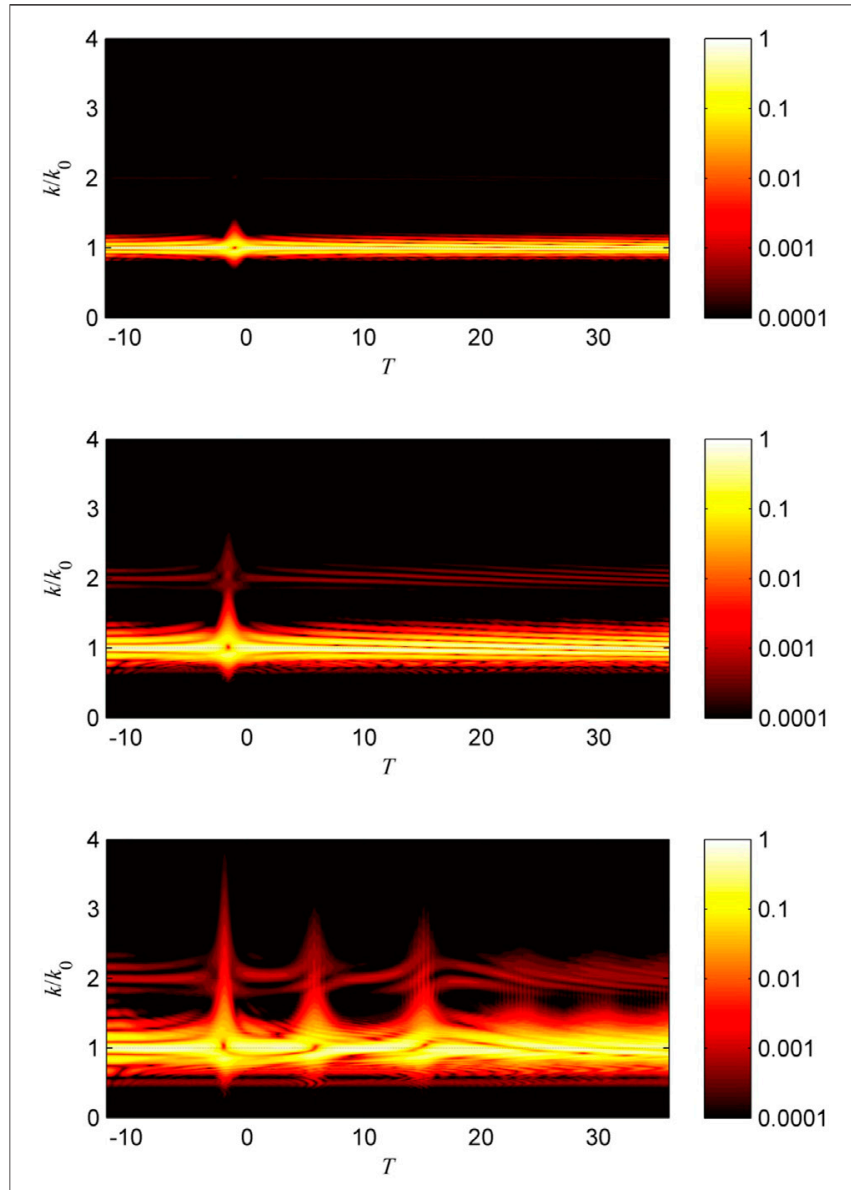

FIGURE 9 | Evolution of the spatial Fourier transform in the cases $k a=$ 0.05 (A), $k a=0.10$ (B) and $k a=0.15$ (C) as in Figure 6. The decimal logarithm of the normalized Fourier power is in the range from 1 down to $10^{-4}$.

NLSE in the description of extreme wave events for a wide range of reasonable carrier wave parameters in nonlinear dispersive wave guides. Moreover, we anticipate motivated studies related to further exploration of such distinct degenerate soliton dynamics in optics and quantum physics. We also believe that the degenerate soliton solution may play a substantial role in the spontaneous formation [42, 43] identification of

\section{REFERENCES}

1. Peregrine DH. Water Waves, Nonlinear Schrödinger Equations and Their Solutions. J Aust Math Soc Ser B, Appl. Math (1983) 25:16-43. doi:10.1017/ s0334270000003891

2. Shrira VI, and Geogjaev VV. What Makes the Peregrine Soliton So Special as a Prototype of Freak Waves? J Eng Math (2010) 67:11-22. doi:10.1007/s10665009-9347-2

3. Dudley JM, Genty G, Mussot A, Chabchoub A, and Dias F. Rogue Waves and Analogies in Optics and Oceanography. Nat Rev Phys (2019) 1:675-89. doi:10.1038/s42254-019-0100-0

4. Kibler B, Fatome J, Finot C, Millot G, Dias F, Genty G, et al. The Peregrine Soliton in Nonlinear Fibre Optics. Nat Phys (2010) 6:790-5. doi:10.1038/ nphys 1740 doubly-localized extreme wave events using data-driven methods [44]. Future studies will be devoted to higher-order soliton degeneracy beyond the collision of two solitons. This will in our opinion further improve our understanding of the role of integrable systems and nonlinear wave interaction in the formation of rogue waves.

\section{DATA AVAILABILITY STATEMENT}

The raw data supporting the conclusion of this article will be made available by authors, upon reasonable request.

\section{AUTHOR CONTRIBUTIONS}

AC conducted the experiments in the water wave flumes. AS performed the HOSM simulations. All Authors designed the experiments, analysed the data and interpreted results, and took part in writing the manuscript.

\section{FUNDING}

AS was partially supported by Laboratory of Dynamical Systems and Applications NRU HSE, of the Ministry of Science and Higher Education of the Russian Federation Grant 075-152019-1931; and by the Grant 21-55-15008 of the Russian Foundation for Basic Research. BK and JD acknowledge support from the French National Research Agency (EUR EIPHI ANR-17-EURE-0002 and PIA2/ISITE-BFC, ANR-15IDEX-03, "Breathing Light" and "Nextlight" projects, and ANR-20-CE30-0004 "OPTIMAL" project). FD acknowledges support from the European Research Council (ERC-2018-AdG 833125 HIGHWAVE).

\section{ACKNOWLEDGMENTS}

AC acknowledges Zachary Benitez and Theo Gresley-Daines for technical support. AS acknowledges support from the International Visitor Program of The University of Sydney.

5. Chabchoub A, Hoffmann NP, and Akhmediev N. Rogue Wave Observation in a Water Wave Tank. Phys Rev Lett (2011) 106:204502. doi:10.1103/ physrevlett.106.204502

6. Bailung H, Sharma SK, and Nakamura Y. Observation of Peregrine Solitons in a Multicomponent Plasma with Negative Ions. Phys Rev Lett (2011) 107: 255005. doi:10.1103/physrevlett.107.255005

7. Chabchoub A, Hoffmann N, Onorato M, and Akhmediev N. Super Rogue Waves: Observation of a Higher-Order Breather in Water Waves. Phys Rev X (2012) 2:011015. doi:10.1103/physrevx.2.011015

8. Chabchoub A, and Akhmediev N. Observation of Rogue Wave Triplets in Water Waves. Phys Lett A (2013) 377:2590-3. doi:10.1016/ j.physleta.2013.07.027

9. Klein M, Clauss GF, Rajendran S, Guedes Soares C, and Onorato M. Peregrine Breathers as Design Waves for Wave-Structure Interaction. Ocean Eng (2016) 128:199-212. doi:10.1016/j.oceaneng.2016.09.042 
10. Chabchoub A. Tracking Breather Dynamics in Irregular Sea State Conditions. Phys Rev Lett (2016) 117:144103. doi:10.1103/physrevlett.117.144103

11. Randoux S, Suret P, Chabchoub A, Kibler B, and El G. Nonlinear Spectral Analysis of Peregrine Solitons Observed in Optics and in Hydrodynamic Experiments. Phys Rev E (2018) 98:022219. doi:10.1103/ physreve.98.022219

12. Xu G, Hammani K, Chabchoub A, Dudley JM, Kibler B, and Finot C. Phase Evolution of Peregrine-like Breathers in Optics and Hydrodynamics. Phys Rev E (2019) 99:012207. doi:10.1103/physreve.99.012207

13. Chabchoub A, Mozumi K, Hoffmann N, Babanin AV, Toffoli A, Steer JN, et al. Directional Soliton and Breather Beams. Proc Natl Acad Sci USA (2019) 116: 9759-63. doi:10.1073/pnas.1821970116

14. Michel G, Bonnefoy F, Ducrozet G, Prabhudesai G, Cazaubiel A, Copie F, et al. Emergence of Peregrine Solitons in Integrable Turbulence of Deep Water Gravity Waves. Phys Rev Fluids (2020) 5:082801. doi:10.1103/physrevfluids.5.082801

15. Kedziora DJ, Ankiewicz A, and Akhmediev N. Rogue Waves and Solitons on a Cnoidal Background. Eur Phys J Spec Top (2014) 223:43-62. doi:10.1140/epjst/ e2014-02083-4

16. Dysthe KB, and Trulsen K. Note on Breather Type Solutions of the NLS as Models for Freak-Waves. Physica Scripta (1999) T82:48. doi:10.1238/ physica.topical.082a00048

17. Magnani M, Onorato M, Gunn D, Rudman M, Kibler B, Akhmediev N, et al. Experimental Realization of Periodic Deep-Water Wave Envelopes with and without Dissipation. Water Waves (2019) 2:113-22. doi:10.1007/s42286-01900015-8

18. Chen J, and Pelinovsky DE. Rogue Periodic Waves of the Focusing Nonlinear Schrödinger Equation. Proc R Soc A (2018) 474:20170814. doi:10.1098/ rspa.2017.0814

19. Akhmediev N, and Ankiewicz A. Spatial Soliton X-Junctions and Couplers. Opt Commun (1993) 100:186-92. doi:10.1016/0030-4018(93)90577-r

20. Zakharov VE. Stability of Periodic Waves of Finite Amplitude on the Surface of a Deep Fluid. J Appl Mech Tech Phys (1968) 9:190-4. doi:10.1007/bf00913182

21. Zakharov VE, and Shabat AB. Exact Theory of Two-Dimensional SelfFocusing and One-Dimensional Self-Modulation of Waves in Nonlinear media. Sov Phys JETP (1972) 34:62-9.

22. Yuen HC, and Lake BM. Nonlinear Dynamics of Deep-Water Gravity Waves. Adv Appl Mech (1982) 22:67-229. doi:10.1016/s0065-2156(08)70066-8

23. Slunyaev A, Clauss GF, Klein M, and Onorato M. Simulations and Experiments of Short Intense Envelope Solitons of Surface Water Waves. Phys Fluids (2013) 25:067105. doi:10.1063/1.4811493

24. Slunyaev A, Klein M, and Clauss GF. Laboratory and Numerical Study of Intense Envelope Solitons of Water Waves: Generation, Reflection from a wall and Collisions. Phys Fluids (2017) 29:047103. doi:10.1063/1.4979524

25. Ablowitz MJ, Kaup DJ, Newell AC, and Segur H. The Inverse Scattering Transform-Fourier Analysis for Nonlinear Problems. Stud Appl Math (1974) 53:249-315. doi:10.1002/sapm1974534249

26. Osborne AR. Nonlinear Ocean Wave and the Inverse Scattering Transform. Scattering (2010) 637-66. Academic Press. doi:10.1016/b978-012613760-6/50033-4

27. Akhmediev N, and Ankiewicz A. Solitons: Nonlinear Pulses and Beams. Springer (1997).

28. Zakharov VE, and Gelash AA. Nonlinear Stage of Modulation Instability. Phys Rev Lett (2013) 111:054101. doi:10.1103/physrevlett.111.054101

29. Satsuma J, and Yajima N. B. Initial Value Problems of One-Dimensional SelfModulation of Nonlinear Waves in Dispersive media. Prog Theor Phys Suppl (1974) 55:284-306. doi:10.1143/ptps.55.284

30. Dudley JM, and Genty G. Supercontinuum Light. Phys Today (2013) 66:29-34. doi:10.1063/pt.3.2045

31. Chabchoub A, Hoffmann N, Onorato M, Genty G, Dudley JM, and Akhmediev N. Hydrodynamic Supercontinuum. Phys Rev Lett (2013) 111:054104. doi:10.1103/physrevlett.111.054104
32. Tikan A, Billet C, El G, Tovbis A, Bertola M, Sylvestre T, et al. Universality of the Peregrine Soliton in the Focusing Dynamics of the Cubic Nonlinear Schrödinger Equation. Phys Rev Lett (2017) 119:033901. doi:10.1103/ physrevlett.119.033901

33. Xu G, Chabchoub A, Pelinovsky DE, and Kibler B. Observation of Modulation Instability and Rogue Breathers on Stationary Periodic Waves. Phys Rev Res (2020) 2:033528. doi:10.1103/physrevresearch.2.033528

34. Chabchoub A, and Grimshaw R. The Hydrodynamic Nonlinear Schrödinger Equation: Space and Time. Fluids (2016) 1:23. doi:10.3390/ fluids 1030023

35. Gomel A, Chabchoub A, Brunetti M, Trillo S, Kasparian J, and Armaroli A. Stabilization of Extreme Wave Events by Phase Space Manipulation. Phys Rev Lett (2021) 126:174501. doi:10.1103/physrevlett.126.174501

36. Dysthe KB. Note on a Modification to the Nonlinear Schrödinger Equation for Application to Deep Water Waves. Proc R Soc Lond A. Math Phys Sci (1979) 369:105-14. doi:10.1098/rspa.1979.0154

37. Slunyaev A, Pelinovsky E, Sergeeva A, Chabchoub A, Hoffmann N, Onorato $M$, et al. Super-rogue Waves in Simulations Based on Weakly Nonlinear and Fully Nonlinear Hydrodynamic Equations. Phys Rev E (2013) 88:012909. doi:10.1103/physreve.88.012909

38. Shemer L, and Alperovich L. Peregrine Breather Revisited. Phys Fluids (2013) 25:051701. doi:10.1063/1.4807055

39. Waseda T, Fujimoto W, and Chabchoub A. On the Asymmetric Spectral Broadening of a Hydrodynamic Modulated Wave Train in the Optical Regime. Fluids (2019) 4:84. doi:10.3390/fluids4020084

40. West BJ, Brueckner KA, Janda RS, Milder DM, and Milton RL. A New Numerical Method for Surface Hydrodynamics. J Geophys Res (1987) 92: 11803-24. doi:10.1029/jc092ic11p11803

41. Slunyaev A, Pelinovsky E, and Guedes Soares C. Reconstruction of Extreme Events through Numerical Simulations. J Offshore Mech Arctic Eng (2014) 136: 011302. doi:10.1115/1.4025545

42. Suret P, El Koussaifi R, Tikan A, Evain C, Randoux S, Szwaj C, et al. Single-shot Observation of Optical Rogue Waves in Integrable Turbulence Using Time Microscopy. Nat Commun (2016) 7:1-8. doi:10.1038/ncomms13136

43. Närhi M, Wetzel B, Billet C, Toenger S, Sylvestre T, Merolla JM, et al. Real-time Measurements of Spontaneous Breathers and Rogue Wave Events in Optical Fibre Modulation Instability. Nat Commun (2016) 7:1-9. doi:10.1038/ ncomms 13675

44. Salmela L, Tsipinakis N, Foi A, Billet C, Dudley JM, and Genty G. Predicting Ultrafast Nonlinear Dynamics in Fibre Optics with a Recurrent Neural Network. Nat Machine Intelligence (2021) 3:344-54. doi:10.1038/s42256021-00297-z

Conflict of Interest: The authors declare that the research was conducted in the absence of any commercial or financial relationships that could be construed as a potential conflict of interest.

Publisher's Note: All claims expressed in this article are solely those of the authors and do not necessarily represent those of their affiliated organizations, or those of the publisher, the editors and the reviewers. Any product that may be evaluated in this article, or claim that may be made by its manufacturer, is not guaranteed or endorsed by the publisher.

Copyright $\odot 2021$ Chabchoub, Slunyaev, Hoffmann, Dias, Kibler, Genty, Dudley and Akhmediev. This is an open-access article distributed under the terms of the Creative Commons Attribution License (CC BY). The use, distribution or reproduction in other forums is permitted, provided the original author (s) and the copyright owner(s) are credited and that the original publication in this journal is cited, in accordance with accepted academic practice. No use, distribution or reproduction is permitted which does not comply with these terms. 